\title{
Direct recovery of lipase derived from Burkholderia cepacia in recycling aqueous two- phase flotation
}

\begin{abstract}
In this paper, aqueous two-phase flotation (ATPF) composed of thermo-sensitive ethylene oxideï propylene oxide (EOPO) copolymer and ammonium sulfate was developed for direct recovery of Burkholderia cepacia (B. cepacia) strains ST8 lipase from fermentation broth. The effect of varying polymer molar mass, concentration of ammonium sulfate, $\mathrm{pH}$, amount of loaded crude feedstock, initial volume of EOPO phase, concentration of EOPO, initial volume of aqueous phase, nitrogen flow rate and flotation time upon ATPF performance were investigated. Under the optimal conditions of ATPF, the average separation efficiency and purification fold are $76 \%$ and $13 \%$, respectively. The recycling of phase components was introduced to minimize the use of organic solvent and salt in ATPF. It was demonstrated EOPO phase in the ATPF system was recovered up to $75 \%$. There was no significant difference in selectivity, purification fold, separation efficiency and recovery yield of lipase obtained between ATPF using fresh and recycled chemicals. B. cepacia lipase was successfully purified by using ATPF, which is composed of copolymer EOPO/ammonium sulfate in a single downstream processing step.
\end{abstract}

Keyword: Lipase; Aqueous two-phase flotation (ATPF); Thermoseparation; Purification; Recycling aqueous two-phase flotation 